\title{
Improving target identification using pairs of composite faces constructed by the same person
}

\author{
C.D. Frowd ${ }^{1}$, V. Bruce ${ }^{2}$, Y. Plenderleith ${ }^{1}$ and P.J.B. Hancock ${ }^{1}$ \\ ${ }^{1}$ Psychology Department, Stirling University, Stirling. Fax: (01786) 467641. Email: cdf1 @ stir.ac.uk. \\ ${ }^{2}$ College of Humanities and Social Science, Edinburgh University, Edinburgh
}

Keywords: facial composite, witness, practice, colour, EvoFIT.

\begin{abstract}
Facial composites produced using traditional feature-based systems are notoriously hard to recognise. We have been developing a new more recognition-based system called EvoFIT that is performing better than other computerised approaches. In the current work, potential ways of improving performance even further were explored. It was found that asking the same person to construct two composites of a target face was successful in improving target identification. The data also found that composites constructed second were as identifiable as those constructed first, suggesting that the system does not appear to be interfering with a user's memory of a target face. The work also indicated that switching from a monochrome to a colour face model produced a slight decrement in performance. Lastly, the work replicated a previous finding that constructing a composite of a distinctive face produces a more identifiable rendition than a composite of a more average-looking face.
\end{abstract}

\section{Introduction}

Witnesses of serious crime are often asked to construct a facial composite, a visual likeness of a suspect. There are three broad approaches available for their production: sketch artists, who use pencils or crayons to draw the face by hand; computerised 'feature' systems, such as E-FIT and PRO-fit in the UK, where witnesses select individual facial features from a kit of parts; and so-called third generation recognition-based systems, such as EigenFIT or EvoFIT in the UK, where witnesses select complete faces from an array of alternatives and a composite is 'evolved' over time (see $[7,16]$ for a more detailed summary of the systems). When working from a photograph of a face, essentially a copying exercise, all approaches appear to produce good likenesses, but this situation changes when construction relies on a person's memory of a face. For the feature-based systems, research in several laboratories report that when participant-witnesses attempt construction within a few hours of inspecting a target face, their composites are named only about $20 \%$ of the time $[2,3,8,13,14]$. Performance falls off even further following a longer delay $[14,15,24]$ and, in this case, both sketch artists and EvoFIT have been found to be somewhat better than UK feature systems $[14,15]$.
There have been several recent attempts to improve the quality of composites. These can be broadly divided into studies that have changed the technology [14,17], improved the procedures used at construction [18], or involved the production of more than one composite of the same target $[1,3]$. This final pair of studies has demonstrated that target identification can be improved if different 'witnesses' construct a composite of the same face. In such a situation, the composites produced may be presented together for identification or combined into a single morphed image. The current work explores whether an identification benefit may be similarly observed if the same person constructs more than one composite.

Computerised systems like E-FIT and PRO-fit are deterministic in nature, and so repeatedly using the same system is likely to produce the same composite. However, faces within EvoFIT start as random points within a multidimensional face space [11,17]. The witness selects those faces closest to the target, which are then bred together to form a new generation. By repeatedly selecting and breeding, the faces gravitate towards the desired target. As the initial faces are random, and the face space very large, repeated use produces different-looking composites. In spite of this inherent variability in performance, EvoFIT composites have been found to be more identifiable than UK contemporary computerised feature systems [16,17]. We argue that asking a witness to construct more than one composite with EvoFIT is therefore likely to improve the chances of producing a recognisable rendition.

Research and development on EvoFIT to date has been carried out using a face model in monochrome. However, the two other recognition-based approaches that we are aware of, EigenFIT [19] and ID [29], are colour-based; there has also been a promising attempt to produce an early feature-based system in colour for the UK [5]. Might it be beneficial for EvoFIT to follow suit? In general, research suggests that while the recognition of faces in colour and monochrome is very similar [6,23,27], a benefit of colour has been found for briefly presented familiar faces [26] and for the recall (descriptions) of faces [6]. As EvoFIT appears to contain an element of both recall and recognition [14,17], and since colour may provide an additional cue to facilitate a less than accurate representation (i.e. a composite), colour may be a useful modality to consider for EvoFIT. 
In the following sections, we describe a recent study which employs EvoFIT to construct more than one composite of the same target face by the same person. It was expected that such multiple attempts would serve to improve the identification of the target face. The work also compared a colour and a monochrome version of EvoFIT.

\section{The EvoFIT composite system}

At the heart of the EvoFIT system is a face generator, a software module that can produce a large number of realisticlooking human faces $[11,17,20]$. The face generator was built using Principal Components Analysis (PCA) of 72 monochrome photographs of faces taken in a front view and under controlled lighting. Faces were also clean shaven and were not wearing glasses. About 250 coordinate points were first manually located around key facial landmarks of each face. PCA of these coordinate points was then carried out to provide a 'shape' model that describes the shape and position of facial features. Randomisation of the shape coefficients from the PCA allows a face to be generated with random shape properties.

In order to render faces with varying skin tone, the faces in the model were morphed to a standard average shape and their pixel intensities for the inner face region subjected to a second PCA. Randomising these coefficients then provides plausible facial 'textures', which can be morphed to a random shape given by the shape model. Note that some approaches for generating a PCA face model explicitly combine shape and texture information, for example $[4,19]$. In practice, witnesses select a set of external features - hair, face shape and neck - at the start of the session and the texture is blended into this template to provide a realistic-looking face. These external features were extracted from the 72 faces used to construct the PCA model.

For the current project, a texture model was also constructed in colour. Three separate PCA models were run on colour versions of the 72 image face set for the red, blue and green channels that make up a colour image. Generation of faces thus involved three sets of texture PCA coefficients and, while production of each face was slower by about $60 \%$, performance on a modern PC was sufficient for a screen of 18 faces to be produced in about 5-10 seconds.

To construct a composite with EvoFIT, witnesses first select a set of external features, as mentioned above, to be shown on all faces. Witnesses are then presented with a series of arrays each containing 18 faces. They are initially shown 72 face shapes, and select six; 72 face textures, and select six; and then choose the best combination of these preferred shapes and textures. After identifying the best overall likeness, the chosen faces are bred together to produce more faces, and witnesses similarly select from this set. Repeated three or four times, the faces become more similar to each other and more similar to the target. Ultimately, the face with the best likeness to the target is saved to disk as the 'composite'. The software also has the ability to improve the likeness of the best face on demand, by allowing shape changes such as making the eyebrows bigger or moving the mouth closer to the nose. Note that, in an attempt to maintain a good representation of the target face, users are also asked to form a mental image of it prior to inspecting each set of faces.

\section{Composite production and evaluation}

The aim of the current work was to evaluate the use of colour and the effectiveness of constructing more than one composite of the same target face. To do this, two stages were required: (1) the construction and (2) the evaluation of a set of composites. Famous faces were used as targets in the first part to allow the resulting composites to be evaluated by naming in the second part. The design was within-subjects for both construction attempt (first / second) and image mode (monochrome / colour): one user constructed all four EvoFIT composites for each target.

\subsection{Target set}

Eight good quality colour photographs of well-known celebrities in the UK were located via an extensive search on the Internet. These images were taken in a front view, with a neutral expression and without facial hair or spectacles. The celebrities included sportsmen (David Beckham, Stephen Hendry, Tim Henman and Michael Owen), pop singers (Ronan Keating, Robbie Williams and Will Young), and a TV presenter (Anthony 'Ant' McPartlin).

\subsection{Composite construction}

To construct the composites, an experienced user looked at a photograph of one of the celebrity faces for 1 minute and then constructed four composites using the EvoFIT procedure detailed in section 2 above. The first two used one image modality, colour or monochrome, the second pair the other (the order was fully counterbalanced). In addition, prior to constructing the second pair of composites, the target face was inspected again for 1 minute to provide the same memory conditions as for the first pair. The user also chose which of the two composites produced from each image type represented the best likeness. Each composite took about an hour to complete and four composites were constructed each day of the same target face. Therefore, 32 EvoFIT composites were constructed ( 8 targets $\mathrm{x} 2$ composites $\mathrm{x} 2$ image modes). Example images can be seen in Figure 1.

To allow comparison with a standard feature system, a further set of composites of these target faces was also constructed using the PRO-fit system. This was also carried out by the same user, who first practiced with PRO-fit for a couple of days, the same as for EvoFIT. As before, she looked at each celebrity photograph for 1 minute, then constructed a composite. To do this, the description of each face was used to locate appropriate features within the system, and each of these choices was then resized and repositioned as necessary in order to create the best likeness. This procedure also took about an hour per composite. 


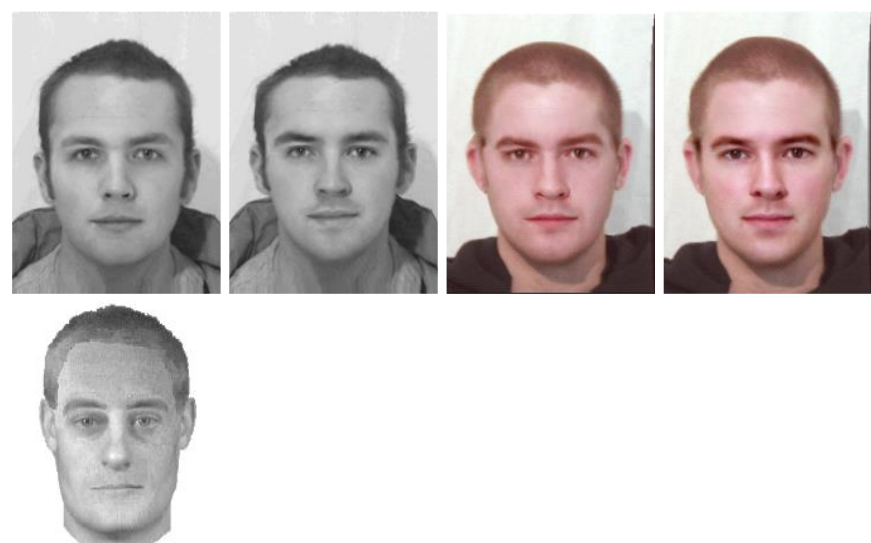

Figure 1. Composites produced of the UK footballer Michael Owen. Along the top row are the EvoFITs (in the order of construction). The first pair of images (on the left) were constructed using the normal monochrome face model, the second pair using the colour one. Underneath is the PRO-fit.

\subsection{Composite evaluation}

Three evaluations were carried out on the composites. In 3.3.1, the 40 composites were named individually to allow analyses by image mode and best likeness (which of the pairs was thought best by the user). In 3.3.2, naming of pairs and individual composites was compared. Finally, an analysis by facial distinctiveness is presented in 3.3.3.

\subsubsection{Naming of individual composites}

In this part, the 32 EvoFIT and the eight PRO-fit composites were each printed (at approx. $6 \mathrm{~cm}$ wide $\mathrm{x} 8 \mathrm{~cm}$ high) on a single sheet of A4 paper in either colour or monochrome as appropriate (note that PRO-fit produces only monochrome images). These images were named by 17 participants. Each person was tested individually, told that the composites were constructed of well-known celebrities and asked to provide a name where possible. Participants were also informed to expect more than one composite of the same identity. The composites were then presented sequentially and participants attempted to name them. Once all the composites had been presented, naming was repeated for the target photographs. The order of presentation of composites and targets was randomised for each person.

Naming of the target photographs was very high, at $93.4 \%$ correct, and suggests that participants were appropriately very familiar with the target set. Overall, the EvoFIT composites were correctly named $26.7 \%$ of the time, and this was considerably higher than for the PRO-fit composites, at $1.5 \%$; this increase was significant using a two-tailed pairedsubjects t-test $\left(\mathrm{t}_{16}=11.7, \mathrm{p}<.001\right)$. Therefore, the EvoFITs were of much better quality than the PRO-fits.

An analysis by image mode was conducted next. In addition, to explore whether performance either improved with practice, or declined after having seen many faces, the analysis was extended to explore the quality of composites constructed first and second. This analysis revealed that composites constructed in monochrome (mean, $\mathrm{M}=29.4 \%$ ) were slightly better named than those in colour $(\mathrm{M}=23.9 \%)$, but there was little difference between composites constructed first $(M=27.6 \%)$ and second $(M=25.7 \%)$. The participant data were then subjected to a two-way repeated-measures Analysis of Variance (ANOVA). This indicated that composite quality did not differ significantly by order of construction $\left(\mathrm{F}_{1,16}=0.4, \mathrm{p}>.1\right)$ nor image mode $\left(\mathrm{F}_{1,16}=2.4\right.$, $\mathrm{p}>.1)$; the interaction between these factors was also not significant $\left(\mathrm{F}_{1,16}=1.8, \mathrm{p}>.1\right)$. Therefore, neither image mode nor construction order significantly influenced composite quality (though refer to the Discussion for a re-analysis which suggests that the monochrome model is marginally better).

Finally, the data was partitioned, for each pair of composites, into best and worst groups as judged by the user. Composites in the 'best' group were correctly named at $31.3 \%$ and this was appreciably higher than those in the 'worst' group, at $22.1 \%$; this difference was very large $(\mathrm{d}=0.83)$ and significant using by-subjects $(\mathrm{t} 16=3.2, \mathrm{p}<.01)$ and by-items $\left(t_{15}=2.4, p<.05\right)$ analyses. This suggests that there is benefit for users to construct a pair of composites and to select the one considered to be the best.

\subsubsection{Naming of pairs of composites}

In this part, the identification of individual and pairs of composites was compared. The design was within-subjects for presentation type (individual / pairs) and three booklets were assembled with each containing EvoFITs presented as either an individual composite or as a pair. Each booklet contained a total of 16 individual or pairs of composites; items were rotated around the booklets so that each composite appeared once on its own and once as part of a pair.

Twenty-seven additional participants were randomly assigned, with equal sampling, to one of the three testing booklets and named the composites therein using the procedure of 3.3.1.

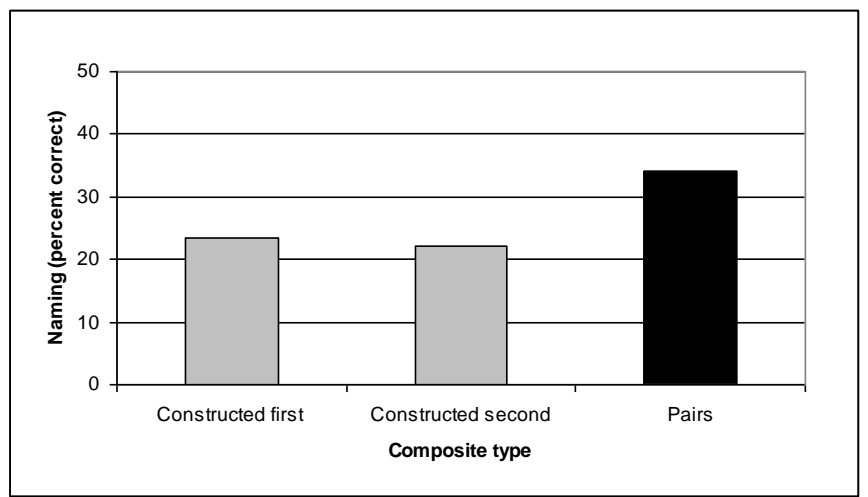

Figure 2. Improving target identification by displaying pairs of composites.

Naming of the target photographs was as before very high, at 91.7\% correct. As illustrated in Figure 2, composites shown on their own, either those constructed first $(\mathrm{M}=24.3 \%)$ or second $(\mathrm{M}=22.9 \%)$, were named similarly to 3.3.1. 
However, presenting composites as pairs substantially improved performance $(\mathrm{M}=35.4 \%, \mathrm{~d}>0.72)$. An ANOVA was significant for composite type $\left(\mathrm{F}_{2,50}=7.8, \mathrm{p}=.001\right)$ and simple-contrasts confirmed the equivalence of the individual composites $(p>.1)$ and the superiority of the pairs $(p<.005)$. Thus, there is a clear advantage for presenting pairs of composites for identification as opposed to an individual rendition.

\subsubsection{Distinctiveness}

An additional analysis was carried out to explore whether target distinctiveness might influence composite quality. It is established in the literature - for example [28] - that face recognition is facilitated when faces are unusual or distinctive in appearance relative to more average-looking examples. Similarly, a recent study also confirmed that composites were better named when constructed of a more distinctive target [16].

For the current work, the eight target photographs were given to a further group of 15 participants for them to rate for distinctiveness $(1=$ average $/ 7=$ very distinctive $)$. This enabled the naming data to be grouped into those constructed of a distinctive target (David Beckham, Ronan Keating, Anthony McPartlin and Robbie Williams; $M=5.7$ ) and those of an average one (Stephen Hendry, Tim Henman, Michael Owen, Will Young; $\mathrm{M}=2.9$ ).

The naming data strongly favoured composites of highly distinctive faces $(\mathrm{M}=40.1 \%)$ over those of average targets $(\mathrm{M}=13.2 \%)$, and the effect size was very large $(\mathrm{d}=1.22)$. An ANOVA confirmed the superiority for composites of distinctive targets $\left(\mathrm{F}_{1,16}=78.4, \mathrm{p}<.001\right)$ and also that this effect was consistent in both monochrome and colour $\left(\mathrm{F}_{1,16}=\right.$ $1.2, \mathrm{p}>.1)$. A similar effect was not found for the PRO-fits, arguably due to low overall naming rates $\left(\mathrm{t}_{16}=1.5, \mathrm{p}>.1\right)$. Therefore, EvoFIT composites again appear to be superior when a target face is distinctive.

\subsection{Discussion}

EvoFIT differs from traditional feature-based systems in that a different composite is produced each time the system is employed. This situation emerges due to the inherent randomness of the procedure. While this may seem a little risky for such a system to be used with real witnesses and victims, the data collected so far $[16,17]$ suggests that a more identifiable composite is produced compared with the more traditional approaches.

In the current work, we explored whether further benefit may be observed if more than one composite is constructed of the same target face, or if colour was used instead of monochrome. These possibilities were investigated by an experienced system user looking at a photograph of a famous face and constructing a pair of composites with EvoFIT, first in one image mode and then the other. The work found that composites constructed first were named statistically the same as those constructed second, but that presenting either both composites together, or just the one judged better by the user, produced similar improved recognition. Also it was found that composites constructed in colour were named statistically the same as composites constructed in monochrome, and that target distinctiveness was an important factor.

An important finding from the study was that constructing two composites with EvoFIT was beneficial. Naming between presenting pairs of composites $(\mathrm{M}=35.4 \%)$ was slightly higher than presenting the single composite that the user thought best $(\mathrm{M}=31.3 \%)$; these data also approach significance $\left(t_{15}=1.7, p=.1\right)$. Interestingly, the correlation is very high between these data $\left(r_{42}=0.94, p<.001\right)$, further suggesting that the benefit of presenting both images derives mainly from the presence of the best. In practice, it may be better to publish a single image for identification in the media as this is the current police procedure.

The construction of a composite with this system involves searching two PCA face spaces (i.e. shape and texture) using a small number of initially random points. Due to the complexity of the search space, a different solution is produced each time, and clearly some of these solutions are better than others. The work has also found that it is possible for a user to identify which composite produced is likely to be more identifiable, even after having perceived about 1000 faces, which is the norm for constructing two composites with EvoFIT. Current work is looking into reducing the complexity of the face space, by using smaller more targetspecific face models, which should further improve the effectiveness of locating a good likeness.

A second key finding was that the use of colour did not improve target identification. However, if the naming data collected for both the individual (section 3.3.1) and pairs of composites (section 3.3.2) are combined to increase statistical power, there is weak evidence in favour of the monochrome format $\left(\mathrm{t}_{42}=1.8, \mathrm{p}<.1\right)$ with a medium level effect size $(\mathrm{d}=$ 0.42 ). Therefore, it would appear best to continue using the monochrome version of EvoFIT.

Why then should a colour version of EvoFIT produce a less identifiable composite than a monochrome version? The design attempted to build a colour face model that was as similar to the monochrome one as far as possible (including using the same faces); and the construction of the composites was the same in monochrome and in colour. Indeed, the established literature suggests in general that performance would be, at worst, the same across image formats (for example [6]). We now believe that rather than providing additional cues for identification, colour is in fact adding an extra layer of complexity. In other words, colour is increasing the complexity of the search space and making it more difficult to locate an identifiable representation!

Such a notion resonates well with current work that has sought to increase the range of faces that may be constructed with EvoFIT [13]. This has involved photographing a wider 
range of faces to enable the development of new models. However, the performance of the new models appears to be somewhat worse than the monochrome version used here. It turns out that the new faces were photographed with greater care, and the shape model refined somewhat to improve image clarity, and so the quality of the resulting face models were better. Consequently, we appear to have unwittingly increased the complexity of the search problem and decreased performance. We are actively exploring ways to overcome this effect: one possibility might be to actually reduce the image quality, perhaps by simply turning down the image contrast level and thereby make the faces appear a little blander.

A third finding was that performance was very similar for composites constructed first and second. If one also combines data across experiments, the same as above for image mode, one still finds that construction order does not influence composite quality $\left(\mathrm{t}_{42}=1.0, \mathrm{p}>.1\right)$. Therefore, there does not appear to be either a practice effect with EvoFIT, where a user might get better constructing the same target face, nor a decrement in performance due to interference caused by the construction process. It turns out that about 500 faces are presented during the construction of an EvoFIT and so one might expect a user's memory of a target might suffer interference (for example [9]). However, given that naming did not significantly change for composites constructed first or second, it would appear that such interference was not an issue. We believe that such interference may be avoided, at least in part, since users are asked to form a mental image of the target at regular intervals throughout construction.

A final key finding was that EvoFIT was able to produce considerably more identifiable composites for targets rated consistently as distinctive: there was a three fold improvement in naming. Past research has found a distinctiveness effect for a range of composite systems [16], including an early version of EvoFIT. While the performance of EvoFIT has improved considerably in the past couple of years, a distinctiveness effect remains.

We acknowledge that the target faces used in this study were familiar to the user, and that there was also no delay to construction, and so the composites were not constructed as realistically as possible in the laboratory. This design was chosen to elevate naming above floor levels, which tend to be quite low with longer delays, and to allow appropriate statistical analyses. In spite of this, the data appear to be sensible, with naming levels overall equivalent (or even a little higher) to those found in similar designs $[2,3,8,13,14]$; there is also a distinctiveness effect. The use of famous faces might especially affect the finding that second composites are as good as first ones, since a famous face will already have an internal representation and should therefore stay in the memory better than an unfamiliar one. We note that ongoing work with a more realistic design (i.e. unfamiliar faces, a 2 day delay and different participant-witnesses) has also found that composites constructed second were of similar quality to those constructed first.
In summary, the current work has demonstrated that target identification can be improved by allowing a constructor to produce more than one composite with EvoFIT. The work has also shown that it is somewhat better to construct in monochrome rather than in colour, a result that designers of the other recognition-based systems might find useful.

\section{Acknowledgements}

This work was funded by a grant from the Engineering and Physical Sciences Research Council, EP/C522893/1. The authors would like to thank Jane Hendry for her assistance with composite production and evaluation, and Cindy and David Frowd for useful feedback on an early draft of the paper.

\section{References}

[1] P. Bennett, "The use of multiple composites in suspect identification", Proceedings of the 3rd UK national conference on cranio-facial identification, (2000).

[2] N. Brace, G. Pike and R. Kemp, "Investigating E-FIT using famous faces". In A. Czerederecka, T. JaskiewiczObydzinska \& J. Wojcikiewicz (Eds.). Forensic Psychology and Law. Krakow: Institute of Forensic Research Publishers, pp. 272-276, (2000).

[3] V. Bruce, H. Ness, P.J.B. Hancock, C. Newman and J. and Rarity, "Four heads are better than one. Combining face composites yields improvements in face likeness", Journal of Applied Psychology, 87, pp. 894-902, (2002).

[4]T.F. Cootes, K.N. Walker, and C.J. Taylor, "View-Based Active Appearance Models", Proceedings of the International Conference on Face and Gesture Recognition, pp. 227-232, (2000).

[5] Davies, G.M. Recall and reconstruction of faces: Implications for theory and practice. In Ellis, H. D., Jeaves, M., Newcome, F. \& Young, A. (Eds.) Aspects of Face Processing, Dodrecht, The Netherlands, Martinus Nijhoff, (1986).

[6] G.M. Davies and S. Thasen, "Closed-circuit television: How effective an identification aid? British Journal of Psychology, 9, pp. 411-426, (2000).

[7] G.M. Davies and T. Valentine, "Facial composites: forensic utility and psychological research". In: R.C.L. Linsay, D.F. Ross, J.D. Read and M. P. Toglia Handbook of eyewitness psychology, Vol. 2: Memory for people. Mahwah: LEA, (in press).

[8] G.M. Davies, P. van der Willik, and L.J. Morrison, "Facial Composite Production: A Comparison of Mechanical and Computer-Driven Systems", Journal of Applied Psychology, 85, pp. 119-124, (2000). 
[9] J. Dysart, R.C.L. Lindsay, R. Hammond and P. Dupuis, "Mug shot exposure prior to lineup identification: interference, transference, and commitment effects", Journal of Applied Psychology, 86, pp. 1280-1284, (2001).

[10] H.D. Ellis. "Practice aspects of face memory", in G. R. Wells and E.F. Loftus, editors, Eyewitness Testimony. Psychological Perspectives. Cambridge, England: Cambridge University Press, Ch. 2., (1984).

[11] C.D. Frowd, "EvoFIT: A Holistic, Evolutionary Facial Imaging System", Unpublished $\mathrm{PhD}$ thesis, University of Stirling, (2002)

[12] C.D. Frowd, V. Bruce, A. McIntyre and P.J.B. Hancock. "The relative importance of external and internal features of facial composites", British Journal of Psychology, (in press).

[13] C.D. Frowd, V. Bruce, A. McIntyre, D. Ross, and P.J.B. Hancock. "Adding Holistic Dimensions to a Facial Composite System", Proceedings of the Seventh International Conference on Automatic Face and Gesture Recognition, Los Alamitos: Ca., pp. 183-188, (2006).

[14] C.D. Frowd, V. Bruce, H. Ness, C. Thomson-Bogner, J. Peterson, A. McIntyre, and P.J.B. Hancock, "Parallel approaches to composite production", Ergonomics, (submitted).

[15] C.D. Frowd, D. Carson, H. Ness, D. McQuiston, J. Richardson, H. Baldwin, and P.J.B. Hancock, "Contemporary composite techniques: the impact of a forensically-relevant target delay", Legal and Criminological Psychology, 10, pp. 63-81, (2005). Also available at http://www.psychology.stir.ac.uk/staff/cfrowd

[16] C.D. Frowd, D. Carson, H. Ness, J. Richardson., L. Morrison, S. McLanaghan, and P.J.B. Hancock, "A forensically valid comparison of facial composite systems", Psychology, Crime and Law, 11, pp. 33-52, (2005).

[17] C.D. Frowd, P.J.B. Hancock, and D. Carson, "EvoFIT: A Holistic, Evolutionary Facial Imaging Technique for Creating Composites", ACM Transactions on Applied Perception (TAP), 1, pp. 1-21, (2004).

[18] C.D. Frowd, D. McQuiston-Surrett, I. Kirkland and P.J.B. Hancock, "The process of facial composite production". In A. Czerederecka, T. JaskiewiczObydzinska, R. Roesch and J. Wojcikiewicz (Eds.). Forensic Psychology and Law. Krakow: Institute of Forensic Research Publishers, pp. 140-152, (2005).

[19] S.J. Gibson, C.J. Solomon, and A. Pallares-Bejarano, "Synthesis of photographic quality facial composites using evolutionary algorithms", Proceedings of the British Machine Vision Conference, Editors R. Harvey and J.A. Bangham, pp. 221-230, (2003).

[20] P.J.B. Hancock, "Evolving faces from principal components", Behav. Res. Meth., Instruments and Computers, 32, pp. 327-333, (2000).

[21] P.J.B. Hancock, V. Bruce, and A.M. Burton, "Recognition of unfamiliar faces", TICS, 4, pp. 330-337, (2000).

[22] P.J.B. Hancock, V. Bruce, and A.M. Burton, "Testing principal component representations for faces", in J.A. Bullinaria, D.W.Glasspool and G. Houghton (Eds.), Proceedings of 4th Neural Computation and Psychology Workshop, London: Springer-Verlag, pp. 84-97, (1997).

[23] R. Kemp, G. Pike, P. White and A. Musselman, "Perception and recognition of normal and negative faces: the role of shape from shading and pigmentation cues", Perception, 25, pp. 37-52, (1996).

[24] C.E. Koehn, and R.P. Fisher, "Constructing facial composites with the Mac-a-Mug Pro system", Psychology, Crime and Law, 3, pp. 215-224, (1997).

[25] K.R. Laughery and M.S. Wogalter, "Forensic applications of facial memory research". In A. Young \& H.D. Ellis (Eds.). Handbook of research on faces. pp. 521-555, (1989).

[26] K.L. Lee and D.I. Perrett, "Presentation-time measures of the effect of manipulations in colour space on discrimination of famous faces", Perception, 26, pp. 733-752, (1997).

[27] D. Perrett, P.J. Benson, J.K. Hietanen, M.W. Oram and D.H. Dittrich, "When is a face not a face?" In R. Gregory, J. Harris, P. Heard \& D. Rose (Eds.). The Artful Eye. Oxford University Press, pp. 99-100, (1995).

[28] P.N. Shapiro and S.D. Penrod, "Meta-analysis of facial identification rates", Psychological Bulletin, 100, pp. 139-156, (1986).

[29] C. Tredoux and Y. Rosenthal, "Face reconstruction using a configural, eigenface-based composite system", Presented at SARMAC III, Boulder, Colorado, July 10, (1999). 